NASA Contractor Report 182266

\title{
Expert Systems Applied to Spacecraft Fire Safety
}

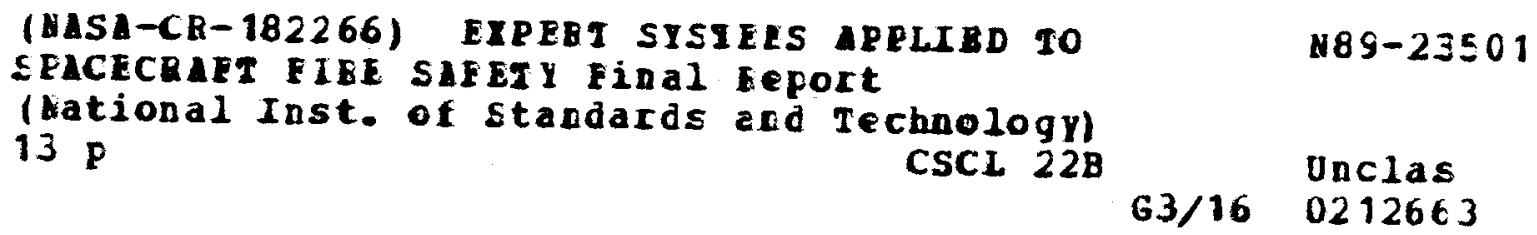

Richard L. Smith and Takashi Kashiwagi

Center for Fire Research

National Institute of Standards and Technology

Gaithersburg, Maryland

June 1989

Prepared for

Lewis Research Center

Under Purchase Order C-32000-M

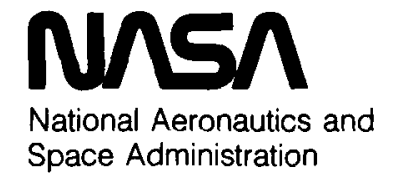




\title{
EXPERT SYSTEMS APPLIED TO SPACECRAFT FIRE SAFETY
}

\author{
Richard L. Smith and Takashi Kashiwagi \\ Center for Fire Research \\ National Institute of Standards and Technology \\ Gaithersburg, Maryland 20899
}

\section{SUMMARY}

Expert systems are problem-solving programs that combine a knowledge base and a reasoning mechanism to simulate a human "expert." The development of an expert system to manage fire safety in spacecraft, in particular the NASA Space Station Freedom, is difficult but clearly advantageous in the long-term. The report discusses some needs in low-gravity flammability characteristics, ventilating-flow effects, fire detection, fire extinguishment, and decision models, all necessary to establish the knowledge base for an expert system.

\section{INTRODUCTION}

This report is a general description of expert-system programs, to describe their unique application in meeting the needs of fire safety in spacecraft, in particular the future NASA Space Station Freedom (refs. 1 to 3).

An expert system is a computer program that solves real-world problems whose solution would normally require a human expert (refs. 4 to 7 ). Assume you are in communication with a fire-safety expert by the use of a terminal. You type in your questions to him; he in return may ask you questions before he gives you his advice. The quality of his advice is what makes him an expert. If you cannot tell whether you are communicating with a human expert or with a computer running an expert system, the computer program qualifies as an expert system. Normally the domain of discourse must be restricted to a particular field of expertise.

This report explains expert systems in terms of their feasibility for development as autonomous intelligence for fire safety in Freedom. A second section of the report discusses the current problems in fire prevention, detection, and control in space, as a preliminary assessment of the presence and absence of expert knowledge in these fields.

In gross terms, an expert system program consists of two major components: a knowledge base and a reasoning mechanism that processes this knowledge to produce additional knowledge. The knowledge base is facts such as the density of iron, the pilot ignition level of dry wood, or the temperature of a circuit board, which are communicated to the knowledge base by a sensor on the circuit board. The reasoning mechanism can be thought of as consisting of two parts: the type of reasoning, either forward or backward reasoning, and the rules that tell what the facts imply. An example may make this clear. Consider the statement "If the chair is combustible and it has contact with a flame for 5 minutes, then it will start to burn." This is what is called a rule. This rule has two "if" conditions and one "then" action or conclusion item. Thus, if the program has this rule as a forward-reasoning rule and the two "if" conditions are in its knowledge base, it will add to the knowledge base the additional fact that the chair will start burning. 
One example of an expert system is MYCIN (ref. 8), which diagnoses and recommends treatment for infectious diseases. In comparisons with medical experts in this limited domain of infectious diseases, the performance of MYCIN is shown to be as good as that of the human experts. Another well-known expert system is DENDRAL (ref. 9), which uses primarily mass spectrographic and nuclear magnetic resonance data to determine the molecular structure of unknown compounds. DENDRAL's performance is superior to most human experts in its domain. Finally, the expert system that is possibly the greatest commercial success is XCON (or Rl as it was originally called) (ref. 10). It is used by Digital Equipment Corporation to configure computer systems for its customers. Because of the large number of possible combinations of computer components, the problem of getting all the components, cables, etc. together to assemble a working system without missing a part or having items left over is very complex. XCON locates all parts in a reasonable arrangement and plans all the connections. It also verifies that the customer order is correct in that there are no missing nor surplus parts. It has been in constant use since 1980, and its performance has been significantly superior to that of human experts.

The main reason to develop an expert system is to make available to a nonexpert the knowledge and expertise of an expert or experts in the most effective manner. Expert systems have a number of unique advantages. A welldesigned expert system will give the user the effect of having a consultant on hand. The simulated consultant will be "blind" in that it cannot make direct observations. However, it will have certain advantages over a human consultant. It will always operate at peak efficiency. It will never have an off day. It will be available 7 days a week, 52 weeks a year. The proposed expert system will have explanation capabilities and infinite patience. It will have the ability to explain all its conclusions and inferences step by step, over and over again. Thus its reasoning will be fully accessible to the program's user. The expert system performance can be made to equal or surpass the performance of most human experts.

\section{EXPERT SISSTEMS FOR SPACE STATION FREEDOM FIRE SAFETY}

\section{Feasibility}

The type of expert system best suited for a space station would be an autonomous system with integrated sensing, computing and controlling functions, installed within the station (ref. 11). The system would monitor sensors in the station, to be informed of the current status of the station as it related to fire safety. It would also have the capability to fight the fires or to take any appropriate action in dealing with a fire. The expert system would thus act as an advisor to humans who are either aboard the station or are elsewhere, recognizing potential fire situations and their control. However, in uninhabited space compartments, the system would operate on its own.

In adapting expert systems to meet fire-safety needs in the future U.S. Space Station Freedom, clearly one must deal with some very difficult problems. There are no human experts recognized as knowledgeable in low-gravity fire conditions, at least comparable to those with expertise in building-fire controls. The development of a fire-safety expert system for Freedom will be slow, costs will be high, and scheduling will be a problem. In addition, the extreme level of required reliability will be an impediment to the development of the system (ref. 12). 
Nevertheless, Freedom has some unique features that encourage the development of an expert system. As a one-of-a-kind assembly, the physical dimensions of the structure and the materials used will be known. Because of the welldefined structure, one can install smart detectors and a fast extinguishing system in the structure. In addition, there is on-going research on expertsystem techniques to provide autonomous operation and maintenance for at least one subsystem in Freedom (ref. 11). The development of a fire-safety expert system can benefit from this research interest and support, and the fire-safety expert system may share existing computer hardware in the space station.

\section{Development Plan}

An expert system is normally developed by first using the expertise of only one expert. This facilitates the evaluation of the performance of the computer program and the collection of knowledge. Later, the expertise of other experts can be added to the program.

A suggested method to develop an expert system for Freedom is shown schematically in Fig. 1. The flow chart steps are, in brief:

(1) Select an expert who will be used as the human expert for the development of the first expert system program.

(2) Select a small consulting group of human experts who will evaluate the performance of the initial program and provide additional expertise so that the program can be improved.

(3) Determine the reasoning methodology of the original expert and produce an initial expert system.

(4) From the previous step, identify serious knowledge gaps that should be filled so the program can perform up to expectations.

(5) Have a group of experts review the previous two items and develop a consensus for a prototype program.

(6) Continue development of the prototype program until its performance is equal to that of the initial expert.

(7) Add the knowledge of the group until the program can perform better than any individual member of the group.

(8) Continue the development of the working system even after it is deployed, by modifying its knowledge base as new knowledge and operating experience are acquired.

\section{FIRE SCIENCE FOR THE SAFETY OF SPACE STATION FREEDOM}

\section{General Overview}

The unique nature of the fire-safety problem in Freedom is discussed in this section. Here we suggest an initial analysis of the fire problem for 
Freedom and the strategy for dealing with fires in the station. This type of analysis would serve as the starting point for an expert system. In the process of developing an expert system, this analysis would be expanded, refined, and modified.

Information on fire behavior in the low-gravity environment of space is very limited, but it is logical to assume that this behavior is significantly different from that commonly observed in normal gravity (ref. 3). While this lack of basic knowledge complicates the study of spacecraft fire safety, in a sense, the fire-safety problem is well defined. As already mentioned, the fire model for Freedom deals with only a few internal geometric configurations compared to the almost limitless numbers for building configurations. Another important feature is that contents and their location are well specified for Freedom compared to buildings. This allows a detailed, scientific fire-safety study of Freedom to be feasible. Also such a study is desirable for the safety of the crew (no possible immediate evacuation from the station) and is cost effective due to the extremely high program costs of Freedom.

Since there is no possibility of immediate escape of the crew from Freedom, the protection of crew members is the essential element of fire safety in the spacecraft. The second necessity is the preservation of the spacecraft structure if a fire occurs. While these two goals are no different than those of fire safety for buildings in a normal-gravity environment, structural preservation is sometimes compromised in buildings in order to control fires. For this reason, the early detection of fire and its subsequent rapid suppression should be the key points of emphasis for the fire safety of Freedom. The crucial requirement is to keep the fire as small as possible and to extinguish it as soon as possible. Suggested approaches to satisfy these requirements are discussed in the next section. If, unfortunately, the fire cannot be contained in an early stage, a drastic measure to suppress the fire is needed, and this approach is also discussed briefly in the next section.

\section{Early Fire Detection in Space}

Various scenarios may be proposed for fires initiated from different sources in Freedom. One such scenario could be a fire initiated from polymeric materials or fluids heated by an accidental local surge in electric power; another could be a fire initiated from an accident in an onboard scientific experiment.

The early detection of a fire caused by an accidental overloading, short circuit, or overheating of an electric device appears to be more difficult in a low-gravity environment than in a normal-gravity environment. The optimum locations of fire detectors are not readily defined in a low-gravity environment due to the lack of a buoyancy-induced upward flow. The flow motion in the spacecraft is determined mainly by a ventilation flow (flow speed of a few centimeters per second) instead of a buoyancy-induced flow. Since the locations and flow rates of vents and the geometrical configuration of the inside of Freedom will be well-specified, calculation of the three-dimensional detailed flow distribution driven by the ventilation inside the modules is feasible, using commercially available engineering codes as a first step (ref. 13). Besides this overall flow calculation, a flow-pattern calculation inside various electronic cabinets, each with its own ventilation, is needed 
to be able to predict flow patterns of early fire products. These flow patterns can be used to predict two important aspects of fire behavior. One is the spatial distribution of fire products in the spacecraft using data on the generation rates of these products; the other is the possible fire growth in an electric cabinet and also in the general space-station volumes using the information on material flammability characteristics. The optimum locations of detectors for early fire detection can be strategically identified from the flow patterns and potential high-risk fire areas. Furthermore, it should be possible to determine the location of fire from the observed pattern of activated detector locations. The complexity of these calculations and the need for real-time response dictate the storing of a library of precomputed and validated results.

The selection of the type of detector sensors is crucial not only to detect fire as early as possible but also to avoid false alarms. The use of a combination of different types of sensitive detectors at various locations might provide useful data as an input to an alarm/no alarm algorithm (rules, decision). The algorithm would contain information from the calculated flow patterns both in the electronic cabinets and in the general spacecraft volumes and it could make a decision whether the detected "fire" is real or false based on the flow pattern applied to the location and type of the activated detectors.

The use of different types of detectors, spatially distributed detectors, and a decision algorithm is aimed at reducing false alarms as much as possible without sacrificing the capability of early fire detection. However, information on fire products is needed for the selection of detector sensors. For example, it is known that an ionization detector is more sensitive to small particulates $(<0.3 \mu \mathrm{m})$ but a light-scattering detector is more sensitive to large particles $(>0.3 \mu \mathrm{m})$. It is not clear what sizes of particulates are formed from a fire in a low-gravity environment. Smoldering, pyrolysis, and localized flaming are possible in the early phase of a fire. In a normalgravity environment, particulates generated from smoldering and pyrolysis tend to be condensed liquids and to be larger than those generated from flaming of the same material. An electrochemical co detector and a flame-ionization detector can be used in combination with a particle-sensitive detector. If quantitative characterization of the generation of the products from smoldering, pyrolysis, and small flames of polymeric materials appropriate for spacecraft is made, the concentration and size distribution of particulates and also concentrations of $\mathrm{CO}$ and other gases can be calculated at various positions in the spacecraft by combining the material-products characteristics with the above flow calculation. The products from smoldering and pyrolysis may not be significantly affected by gravity, but those from small flames might be. Extensive studies are needed to obtain the relationship between the fire products in a low-gravity environment and those in a normal-gravity environment. The calculated concentration distribution in both electronic cabinets and in the general spacecraft volumes will provide detailed information for the selection of the strategic locations of detectors and also for threshold criteria of toxic hazards for the crew. This information can also be used for the determination of the best selection of the crew's evacuation route and method (stay near the floor or float near the ceiling). 
The screening of polymeric materials which are going to be used in Freedom can contribute significantly to the fire safety of Freedom. Although it is generally considered that polymeric materials are less flammable in a lowgravity environment than in a normal-gravity environment (there are limited experimental data, e.g., refs. 14 and 15), more studies are needed to obtain quantitative relationships between the two different gravity environments. As discussed above, knowledge of the characteristics of smoldering, pyrolysis, ignition, and flame spread of the polymeric materials is important in order to predict fire behavior. Thus, low-gravity flammability tests should be used not only for screening of the materials but also to measure quantities useful for predicting fire scenarios. The measured material-flammability characteristics should be stored in the algorithm for early fire detection and fire growth analysis, as described above. Products distribution (particulates, CO, CO2, total hydrocarbons, acutely toxic gases such as HCN, HCL and acrolein) and their generation rates should be measured under smoldering, pyrolyzing, and small flaming modes.

\section{Fire Suppression in Space}

After the detection of a fire, rapid suppression of the fire is essential. As discussed above, an early fire may be in one of the possible modes of smoldering, pyrolyzing, or small flaming for polymeric materials (cables, insulation materials, papers and so on) or fluids. The suppressant must suppress (extinguish) the three types of fire modes. The present Shuttle extinguishing agent, a halogenated hydrocarbon or halon (refs. I and 3), is recognized as an excellent extinguisher for the flaming-fire mode, but it is less effective for the other two types. Furthermore, the use of halons raises questions of the toxic and corrosive nature of their reaction products in flames. A fine water spray is effective for the extinguishment of the flaming-fire mode, but its effectiveness for the other two modes in a low-gravity environment is questionable due to the low sticking efficiency of water droplets onto a smoldering or pyrolyzing surface. Furthermore, the moisture concentration in the atmosphere could become higher with the use of water, which tends to increase corrosivity of metals by fire products, and cleaning of this excess water after the fire might be a problem.

A promising concept for a fire suppressant for space is a fire-extinguishment foam generated with compressed nitrogen. The foam should have a good sticking nature on the surface in a low-gravity environment (however, apparently little is known about the performance of foams in low gravity), and also it is an effective extinguishing agent for all three fire modes. Furthermore, the total mass of liquid is so small that cleaning after the fire is relatively simple. The foam must have a very high dielectric constant to avoid any shorts in electronic devices. Many small cans of the foam extinguishers (like a shaving cream) can be strategically installed at potential fire-hazard areas for use as portable fire extinguishers or as fixed extinguishers to be actuated remotely to fill up the designated compartment. Information regarding the relationship between the amount of the foam and an extinguishable fire size would be needed to test the plausibility of this approach. Since fire behavior at low gravity is poorly understood, its suppression requires imaginative and thorough development. 


\section{Drastic Measures}

If the above fire-fighting tactics fail and a fire becomes large enough to threaten the safety of the crew or the integrity of the spacecraft, some drastic measure is needed to extinguish the fire as quickly as possible. The best approach appears to be venting the module to the outside vacuum of space as described below. Here it is assumed that the module is compartmentalized and can be vacuum tight. It is not necessary to reduce the pressure to vacuum levels but only to a low total pressure sufficient to ensure that the remaining oxygen quantity (partial pressure) in the environment cannot sustain any fire. While inert-gas pressurization has been investigated for extinguishment of difficult fires in confined compartments in submarines (ref. 16), venting to space is much faster than inerting and does not require a large quantity of inert gas storage. Also, when venting is employed, there is no need to provide the extra structural weight required to deal with the problem of over-pressurization of the spacecraft structure. Halons could be also used for this stage of a fire, but there might be some serious reservations due to possible toxic and corrosive natures of halon-flame reacted compounds. Although venting might increase or transfer fire momentarily along the flow to the venting opening (ref. 3 ), the rapid decrease in pressure in the module should suppress the fire quickly. Another advantage of venting is the rapid dilution of harmful by-products in the atmosphere; hence, halons or water may be used for control of difficult fires followed by venting to avoid the deleterious effects of these extinguishants on the atmosphere. One disadvantage of venting is that the vented products may coat the outside surface of windows of the spacecraft with deposits; this might hinder certain scientific experiments. This problem has been experienced with normal waste-product venting and micrometeorite etching in the Soviet space-station program (ref. 17). Potential solutions include retractable transparent covers. Obviously, the consequences and benefits of suppression by venting to vacuum needs a thorough analysis.

Another important decision is under what conditions drastic venting is to be applied. The decision should be based on the safety of the crew and the structural integrity of the space station. Co concentrations, temperatures, pressure, and other properties that relate to the safety and the structural integrity should be considered as indicators to constitute a decision algorithm regarding venting. This algorithm should include possible fire scenarios and calculated potential fire-growth histories based on fuel loading and material flammability characteristics. The approach to a lethal co concentration level or maximum temperature and pressure levels should be used as one criterion for the venting decision in the algorithm. Therefore, the algorithm can be one large code that includes the results of flow-pattern calculations in electronic cabinets and in the general spacecraft volumes, the decision analysis on early fire detection, the collection of CO concentrations, temperatures and other properties, and finally the decision analysis on the actuation of venting.

\section{CONCLUDING REMARKS}

This report explores the capabilities of expert-system programs in their application to fire-safety management in future spacecraft, a new role for these programs. The concept and development of expert systems are briefly described. The goal of this review is the assessment of expert systems for 
autonomous and crew-assisted fire-safety management in the NASA Space Station Freedom, now under design.

Although the development of an expert system for spacecraft fire safety can be very costly and time-consuming, this application has clear advantages in safety operations. What is lacking at present is an adequate knowledge base to simulate that of a human "expert." This report thus includes a discussion of fire-safety problems in spacecraft. From these findings, it is possible to identify some important elements of new knowledge or capabilities needed to establish an expert-system program for Freedom. These items are, in summary:

1. Determination of flammability characteristics of combustible materials in the low-gravity environment of Freedom

2. Development and validation of the ventilating and exhaust-flow calculations in electronic cabinets and in the general module volumes

3. Determination of the generation rate and characteristics of fire products from smoldering, pyrolysis, and small flaming of polymeric materials in a low-gravity environment

4. Development of new detectors, such as a highly selective co detector

5. Development of new fire extinguishers, such as foams, and determination of their properties and extinguishment characteristics

6. Development of an algorithm or algorithms to make the decision as to a false alarm or a real fire from the collection of CO concentrations, temperatures, and other properties, and to make the decision of the venting based on the stored possible fire-growth histories

Like a person entering a new field or discipline, a program on its way to becoming an expert program will start as a novice. If it continues its development, it eventually becomes an expert. Just as an apprentice can help the journeyman, an apprentice expert system will be of significant value, and a journeyman expert system will be of even greater value. Thus we see the evolution of a series of expert systems that will be of great value in their domain of interest to Space Station Freedom. Each of these programs will improve the qual ity of the decision-making of the human expert that it aids.

\section{REFERENCES}

1. Spacecraft Fire Safety. NASA CP-2476, 1987.

2. Rodney, G.A.: Safety Considerations in the Design of Manned Spaceflight Hardware. IAF Paper 87-569, Oct. 1987.

3. Friedman, R.; and Sacksteder, K.R.: Fire Behavior and Risk Analysis in Spacecraft. NASA TM-100944, 1988.

4. Smith, R.L.: ASKBUDJR: A Primitive Expert System for the Evaluation of the Fire Hazard of a Room. NBSIR-86/3319, Mar. 1986. 
5. Weiss, S.M.; and Kulikowski, C.A.: A Practical Guide to Designing Expert Systems. Rowman \& Littlefield Publishers, Totowa, NJ, 1984.

6. Goodal1, A.: The Guide to Expert Systems. Learned Information, Inc., Medford, NJ, 1985.

7. Winston, P.H; and Prendergast, K.A., eds.: The AI Business: Commercial Uses of Artificial Intelligence. MIT Press, 1984.

8. Buchanan, B.; and Shortliffe, E.: Rule based Expert System: The MYCIN Experiments of the Stanford Heuristic Programming Project. Addison-Wesley Publ. Co., Reading, MA, 1984.

9. Lindsay, R.K., et al.: Applications of Artificial Intelligence for Organic Chemistry: The DENDRAL Project. MCGraw-Hill Book Co., 1980.

10. Bachant, J.; and McDermott, J.: Rl Revisited: Four Years in the Trenches. AI Mag., vol. 5, no 3, Fall 1984, pp. 21-32.

11. Lum. H.; and Heer, E.: Progress Toward Autonomous, Intelligent Systems. IAF Paper 87-31, Oct. 1987.

12. Heer, E.; and Lum, H.: Raising the AIQ of the Space Station. Aerosp. Am., vol. 25, no. 1, Jan. 1987, pp. 16-17.

13. Davis, R.G.; and Reuter, J.L.: Intermodule Ventilation Studies for the Space Station. SAE Paper 871428, July 1987.

14. Andracchio, C.R.; and Cochran, T.H.: Gravity Effects on Flame Spreading Over Solid Surfaces. NASA TN D-8228, 1976.

15. Olson, S.L.: The Effect of Microgravity on Flame Spread Over a Thin Fuel. NASA TM-100195, 1987.

16. Tatem, P.A.; Gann, R.G.; and Carhart, H.W.: Pressurization with Nitrogen as an Extinguishant for Fires in Confined Spaces. Combust. Sci. Technol., vol. 7 , no. $5,1973, \mathrm{pp} .213-218$.

17. Feoktistov, K.P.; and Demchenko, E.K.: Description, Photos of Salyut-7 Station. USSR Report: Space, No. 20, JPRS 82970, Joint Publ. Research Service, Arlington VA, Feb. 1983, pp. 8-16 (translation from Zemlya i Vselennaia, No. 6, Nov.-Dec., 1982, pp. 11-16). 


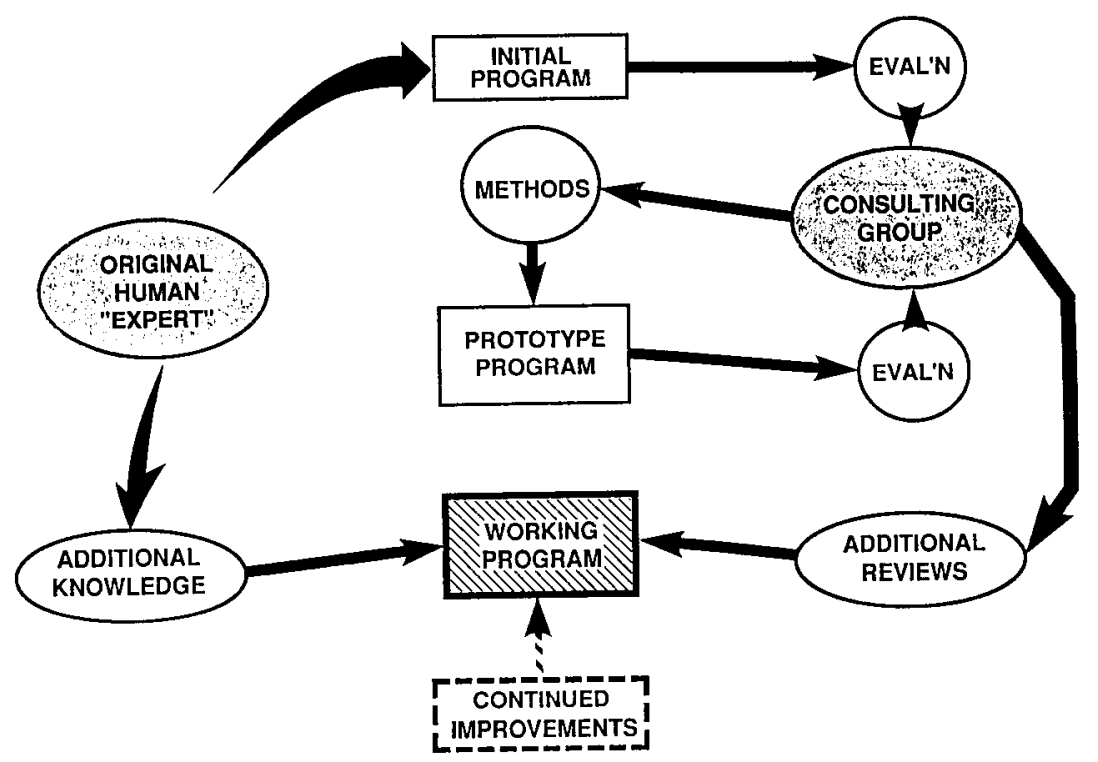

FIGURE 1. - REPRESENTATION OF DEVELOPMENT OF AN EXPERT SYSTEM. 


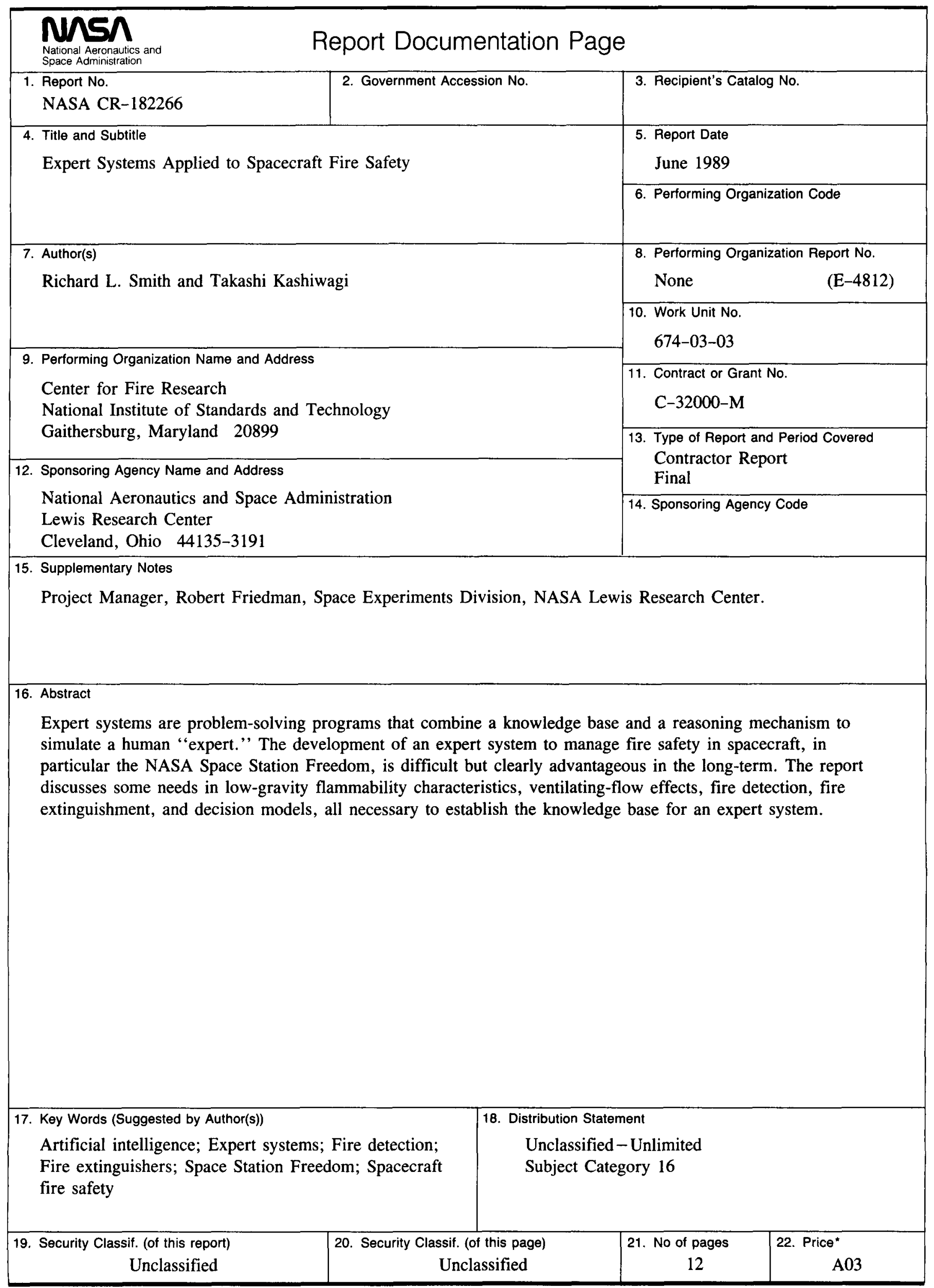

